\title{
151 NUTRITION SUPPORT FOR ELITE ATHLETES: LESSONS LEARNT FROM THE 2006 COMMONWEALTH GAMES
}

Fiona E Pelly University of the Sunshine Coast, Sippy Downs, Australia

10.1136/bjsm.2010.078725.151

Provision of a nutrition support service at elite level competition can assist athletes to make informed food selection at a critical time in their sporting career. Research conducted at the Sydney 2000 Olympic Games revealed that nutrition labelling and access to dietitians was rated highly and widely used by athletes across a range of countries, cultures and sports. The aim of this study was to evaluate a comprehensive nutrition support service within the athletes' village of the Melbourne 2006 Commonwealth Games. The nutrition services available included nutrition labelling of menu items, fact sheets, individual or group dietetic consultations and a request service to cater for athletes with special dietary requirements. Dietitians kept detailed records of each occasion of service from the week prior to until the completion of competition. At the end of each consultation, clients were invited to complete an evaluation of the service provided. In addition, a questionnaire was distributed randomly to patrons in the dining hall. The majority of athletes $(67 \%)$ rated nutrition information as being very important or essential in determining their food choice, with $87 \%$ of athletes using the nutrition labelling at least some of the time. Nearly $60 \%$ of respondents said that the menu met their special dietary needs, supported by the small number $(n=8)$ of requests requiring specialised catering. Evaluation of the nutrition support service confirmed its beneficial role in supporting the needs of athletes at elite level competition but also highlighted areas which could be improved for future games. 